\title{
Saliency Detection by Compactness Diffusion
}

\author{
Qi Zheng \\ qiz@hust.edu.cn \\ Peng Zhang \\ zp_zhg@hust.edu.cn \\ Xinge You \\ youxg@hust.edu.cn
}

\author{
School of Electronic Information and \\ Communications \\ Huazhong University of Science and \\ Technology, \\ Wuhan, P.R. China
}

\begin{abstract}
Most existing methods of salient object segmentation only focus on foreground cues such as contrast, or background cues such as boundary connectivity. Another problem is that they have used redundant information to generate an acceptable saliency map such as variances in different color spaces, multi-scale features and so on. In this paper, we propose saliency detecting with a diffusion model; use optimal seeds generated from foreground statistic cue, i.e., the compactness. Each superpixel is considered as a node and a fully connected graph is constructed to calculate the global compactness of each node. Then the local connected graph is constructed by only considering adjacent nodes, and compactness is diffused by applying a quadratic energy model to generate a coarse saliency map. After that, boundary prior is combined with the coarse saliency map for further eliminating the background. Experiments on three benchmark datasets including MSRA 1000, ECSSD and DUT-OMRON show that compared with other seven stateof-the-art methods, our model achieves stable and excellent performance. Parametric sensitivity analysis and time consumption are given to prove that the proposed method is stable and efficient.
\end{abstract}

\section{Introduction}

With the development of computer science and artificial intelligence, saliency detection has been a hot field especially since recent years. Information obtained from images or video streams is sufficient enough for some tasks such as image matching [0], robot localization [ $\mathbf{\square}]$, automatic collage creation [ $[\mathbf{⿴}]$ and so on. While in high-level real-time tasks, simple but efficient methods are needed as a pre-processing step, which emphasizes the importance of saliency detection [ $⿴ 囗 ⿱ 一 一]$. From the viewpoint of psychology, people are likely to focus on the most different part within the range of vision [ $[$ ] $]$. Based on this consideration, three branches are developed according to [曰], denoted as Visual Attention Modelling (VAM), Salient Object Detection (SOD) and Salient Object Segmentation (SOS) respectively. Among them, we focus on the SOS problem in this paper, which is also called saliency detection in many works [四, 四, 四]. 


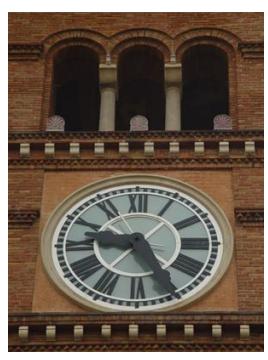

(a)

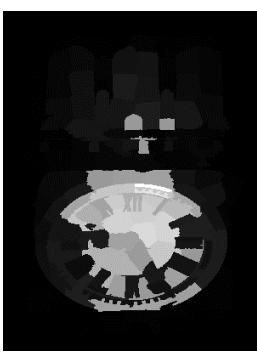

(b)

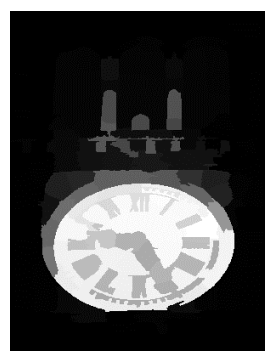

(c)

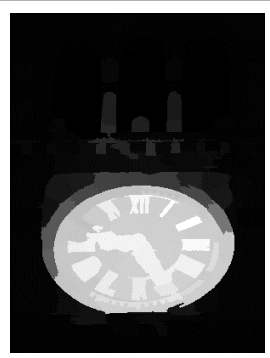

(d)

Figure 1: Hierarchical salient object segmentation through integration of foreground and background. (a) shows a sample image from MSRA 1000 dataset [ $[\mathbb{d}]$. (b) result of our compactness measurement. (c) segmentation result of quadratic energy model with compactness. (d) segmentation result of quadratic energy model after adding boundary prior.

Most of current methods start either from foreground cues or background cues. In foreground-based methods such as contrast-based methods $[\mathbf{\square}, \mathbb{\square}, \mathbf{\square}]$, they extract color features in different color spaces and various texture features from original image, and then measure the differences between patches or regions. In background-based methods such as graph-based methods [ $[\mathbf{D}, \mathbb{Z}, \mathbf{B}]$, they construct a graph with all superpixel-nodes, and set nodes adjacent to image boundary as background, after that they measure the difference between other nodes and background. However, single cue is not always enough to segment salient object in an image. Intuitively, more robust performance can be achieved if independent foreground and background cues are combined, as shown in Fig. 1.

As we consider salient object segmentation as a pre-processing step of more complex tasks, we focus on bottom-up methods that are driven by images themselves. Inspired by diffusion-based models $[\square,[0]$, we integrate independent foreground and background cues with a quadratic energy model. We introduce existing models in Sect. 2. Sect. 3 describes compactness and the quadratic energy model. We show comparative results in Sect. 4. Finally conclusion in Sect. 5. There are three contributions of our work:

1) We propose compactness as an independent cue to extract foreground, and then apply quadratic energy model to diffuse saliency.

2) We analyze the difference between ordinary optimization model for saliency detection and the quadratic energy model.

3) The proposed model has a stable and competitive performance under either simple or complicated background with high efficiency.

\section{Related Work}

Two intuitive ways to segment salient objects includes: 1) find out regions that are most different from other parts in image; 2) suppress repeated patterns or regions to pop out foreground parts. Popular salient object segmentation methods solve this problem either in spatial domain or in frequency domain. Existing methods that model bottom-up, low-level saliency can be roughly divided into the following three categories.

Contrast-based models Contrast-based models include both local and global contrast based

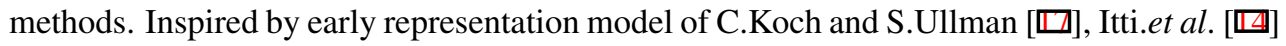
suggested using a set of "center-surround" filters to extract various local contrast including 
color, intensity and orientation in different scales, and saliency map was generated from their linear combinations. Similarly, T. Liu et al. [ $[\mathbf{D}]$ made a linear combination of multiscale contrast to produce pixel-level saliency. In [ $[$ ], M. Cheng et al. proposed a histogram-based contrast method to measure saliency, and improved it with region-based contrast. D. Zhang et al. [B] used superpixel-level contrast to extract intra-saliency. Perazzi et al. [Q⿴囗⿰丿㇄口] defined element uniqueness and element distribution of superpixels according to their contrast and then assign saliency to each superpixel.

Frequency-domain methods As patterns that appear more frequently are more likely to be background, X. Hou et al. []] extracted the spectral residual of an image in spectral domain and constructed the corresponding saliency map in spatial domain. C. Guo et al. []] pointed out that the phase spectrum is the key in calculating the location of salient areas. $\mathrm{J}$. Li et al. [四] proposed hypercomplex Fourier transform and convolved the image amplitude spectral with a low-pass Gaussian kernel to suppress background. Radhakrishna et al. [एव] analyzed spatial frequency content retained in saliency maps of different methods and used DoG band pass filters to find image saliency.

Graph-based models B. Jiang et al. [미 formulated saliency detection via absorbing Markov chain on an image graph model, and then separated background from salient objects according to the absorbed time. C. Yang et al. [四] proposed a graph-based manifold ranking model to detect salient objects with boundary nodes as background seeds. Based on this model, Q. Wang et al. $[\square]$ added connectivity with and within boundary nodes in order to catch global saliency cues. W. Zhu et al. [B] proposed boundary connectivity to measure how likely a region belongs to background, and then solved saliency detection with an optimization model with boundary. K. Chang et al. [] constructed a graph model to integrate objectness and saliency with an energy function, and improved their estimation by iteratively optimization. Y. Wei et al. [ [Q] ] exploited boundary and connectivity as priors and proposed geodesic saliency for object level saliency detection.

Our proposed work concerns graph-based model on superpixel level. We define compactness to find foreground seeds and then use boundary prior as a complement. Thanks to this new measurement, we are able to estimate the number of salient objects so that it improves robustness under different cases, which will be explained in later sections.

\section{Hierarchical Saliency Detection with Quadratic Energy Model}

\subsection{Overall Framework}

Given an image over-segmented by SLIC []], our model aims to assign saliency for each superpixel-node, as shown in Fig. 2. First, we calculate the global compactness of each node and diffuse it to generate coarse saliency. Next, we build a diffusion-based model to extend the coarse saliency according to the local relationships, reflected by adjacency within the graph. Finally, saliency map is integrated with background cue to remove image boundaries effect.

\subsection{Coarse Saliency Map Generation}

As we see, low-level cues from original image such as contrast and texture are not strong enough to produce a perfect saliency map. However, statistics based on these cues can be 


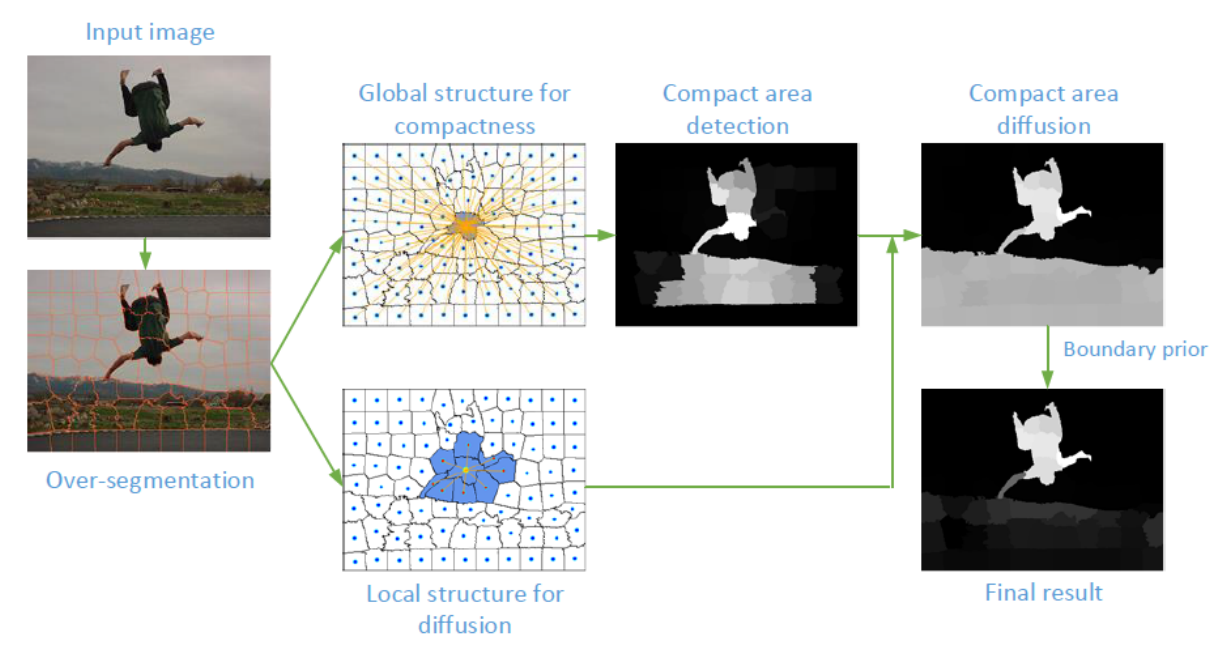

Figure 2: Pipeline of the proposed algorithm, including graph construction, compact area detection, compact area diffusion and boundary cue integration. Nodes in the graph are superpixels.

surprisingly effective, e.g., the color histogram used in [0]. In wireless spectral sensing, false alarm and missing detection are two complemented tools to sense the utility of the channel [ $\mathrm{Q}$, 四]. In domain adaptation, sources from multiple domains are collected to improve final decision $[\square]$. Inspired by these ideas, we consider background and foreground from two independent aspects. On one hand, a widely-used rule is that repeated patterns or colors are more likely to be background $[\square, \square, \square]$, such as grass, sky and so on. However, some parts in foreground may be considered as background in this way, as illustrated in Fig.4, which corresponds to false alarm. On the other hand, salient objects or areas are always in a relatively compact status. As compactness is a global concept so that some small parts in foreground may be missing in detection, which corresponds to miss detection.

Compact Area Detection Given an input image $I$, first we over-segment it with SLIC [ $\square]$ algorithm, thus we derive a series of superpixels $P=\left\{p_{1}, p_{2}, \cdots, p_{n}\right\}$. As shown in Fig. 3, each superpixel is considered as a node so that a fully-connected graph is constructed to detect compact areas globally. The weight of each edge is set to 1. In CIELab space, mean color and mean location are calculated of each superpixel, noted as $\left\{\mathbf{c}_{\mathbf{1}}, \mathbf{c}_{2}, \cdots, \mathbf{c}_{\mathbf{n}}\right\}$ and $\left\{\mathbf{x}_{\mathbf{1}}, \mathbf{x}_{\mathbf{2}}, \cdots, \mathbf{x}_{\mathbf{n}}\right\}$ respectively. Then, the compactness $s_{i}$ of superpixel $p_{i}$ is measured as follows:

$$
\frac{1}{s_{i}}=\sum_{j=1}^{n} \frac{\left\|\mathbf{x}_{\mathbf{j}}-\mathbf{x}_{\mathbf{i}}\right\|^{2}}{\left\|\mathbf{c}_{\mathbf{j}}-\mathbf{c}_{\mathbf{i}}\right\|+\varepsilon}
$$

where $\varepsilon$ is a small value to avoid zero-denominator. It is noticed that, when superpixel $j$ has similar color with superpixel $i$, it is expected that superpixel $j$ is close to superpixel $i$ in space. If lots of superpixels which are similar to superpixel $i$ in color space distribute dispersively among the image, in other words, have a large spatial variance, then we can assume superpixel $i$ belong to background. On the contrary, a small dispersion indicates the compactness of superpixel $i$.

Suppose there is a node located in three different positions $A, B$ and $C$, as illustrated in 
Fig. 3. According to the definition given by Eq. 1, node compactness in position $A$ is the largest, and in position $C$ the smallest.

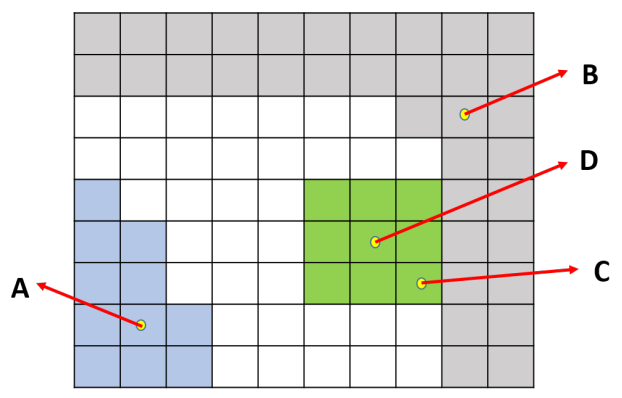

Figure 3: Illustration of compactness: a superpixel-node represented by a grid. Nodes in four different positions $A, B, C$ and $D$ are discussed.

Compactness Diffusion Note that in Fig. 3, compared with position $C$, the node located in position $D$ which has a similar or the same color as it in position $C$, however, because position $D$ is the center of the green area, node $D$ has a grater compactness than node $C$. In order to detect a uniform salient area, a diffusion model is applied to eliminate this in-equality.

Inspired by the idea of [Q]], we set the detected compact areas as optimal seeds, and build a graph-based diffusion model. At this time, a local structure is adopted, in which we only consider adjacent superpixels as shown in Fig. 3. Weight of edge between superpixel $i$ and an adjacent superpixel $j$ is assigned according to the similarity

$$
w_{i j}=e^{-\frac{\left\|c_{i}-c_{j}\right\|^{2}}{\sigma^{2}}}
$$

where $\sigma$ is the standard deviation of all pairs of distance in color space. Then the affinity matrix $W=\left(w_{i j}\right)_{i, j=1,2, \cdots, n}$.

Given compactness value of each node as $\left\{s_{1}, s_{2}, \cdots, s_{n}\right\}$, a diffusion model assigns a saliency value for each node that minimizes the energy function of the form

$$
\mathbf{y}=\arg \min _{\mathbf{y}} \sum_{i}^{n}\left(y_{i}-s_{i}\right)^{2}+\frac{1}{2} \lambda \sum_{i, j} w_{i j}\left(y_{i}-y_{j}\right)^{2}
$$

where $\lambda$ is used to balance the two terms. Note that this model is very similar to CRF models [ $[\square, \mathbb{Z}]$ ], it can be solved by Gaussian edge potentials [ष]]. However, taking into account the efficiency, we choose to solve it with Laplacian graph [ [ $[$ ] $]$ as the same as in [四]. The optimization problem has a closed form solution

$$
\mathbf{y}^{*}=(I+\lambda L)^{-1} \mathbf{s}
$$

where $L$ is the graph Laplacian matrix $[\square]$. The degree matrix $D$ is defined as the diagonal matrix with the degrees $d_{1}, \cdots, d_{n}$ of each node

$$
d_{i}=\sum_{j=1}^{n} w_{i j}
$$

Then the unnormalized graph Laplacian matrix $L=D-W$ [ㅁ]. 


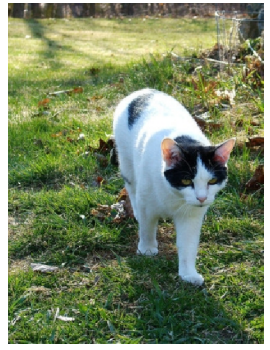

(a)

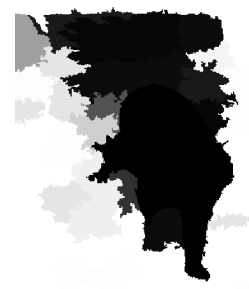

(b)

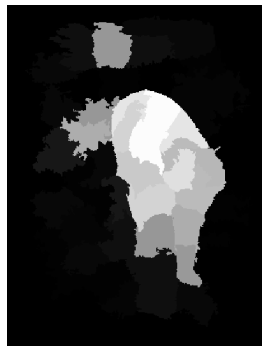

(c)

Figure 4: Conflict detection results by background cue and foreground cue. (a) is an example image from MSRA 1000 [ $\square]$ ]. (b) detected background using boundary connectivity [ $\mathrm{B}$ ]. (c) detected foreground using compactness.

\subsection{Integration with Boundary Prior}

As we mentioned before, the first drawback of compactness is the in-equality, which results in incomplete segmentation. It is eliminated by the diffusion model in Eq. 3. Another drawback is that some parts of background around the center of image may have a relatively high compactness so that it will be considered as foreground, as shown in Fig. 2 (c). To further pop out background, the widely-used boundary prior is integrated with the former result, where nodes adjacent to image boundaries are more likely to be background.

Set $\mathbf{b}=b_{1}, b_{2}, \cdots, b_{n}, b_{i} \in\{0.2,0.8\}$ as the vector that indicates whether a node belongs to background, 0.8 for boundary-adjacency nodes. Integrate boundary prior with former saliency by point-wise multiplication

$$
\mathbf{s}_{b}=\mathbf{y}^{*} \cdot(1-b)
$$

While background is relatively continuous, i.e., as shown in Fig. 3, nodes near boundaries are likely to be part of background. Therefore, we replace the former compact seeds $\mathbf{s}$ with $\mathbf{s}_{\mathbf{b}}$, and diffuse the saliency with the model again

$$
\mathbf{y}_{\dagger}^{*}=\arg \min _{\mathbf{y}_{\dagger}} \sum_{i}^{n}\left(y_{\dagger_{i}}-s_{b i}\right)^{2}+\frac{1}{2} \lambda \sum_{i, j} w_{i j}\left(y_{\dagger_{i}}-y_{\dagger j}\right)^{2}
$$

\subsection{Analysis about Quadratic Energy Model}

Note that in RBD [四], the authors proposed an optimization model similar to our quadratic energy model. Actually, a difference between these two models is that in their optimization model, background and foreground weights simultaneously influence the model, therefore, these two cues sometimes could be conflict, as shown in Fig. 4. The top part is considered as foreground according to background detection $(b)$, while it belongs to background in foreground detection $(c)$. However, in a quadratic energy model, first optimal seeds are chosen, and then the model is applied to diffuse coarse saliency indicated by those seeds. Additionally, boundary connectivity may fail under two cases. The first case is for images that have a frame around image boundary, such as photos with frames, or drawings with frames. The other case is that part of objects is adjacent to image boundary, such as feet. Whereas, our quadratic energy model is able to overcome the negative effect of boundary cue, because the diffusion is applied after its integration with foreground cue. 


\section{Experiments and Results}

To evaluate the performance of our model, we compare it with seven state-of-the-art methods, i.e., MR [四], RBD [四], SF [四], GS [四], HC [四], DSR [四] and DRFI [四] on three public datasets including MSRA 1000 [四], ECSSD [四] and DUT-OMRON [四]. The dataset MSRA1000 contains 1000 images. Although they have a large variety in content, the foreground is always relatively prominent among the whole image. Therefore, we choose ECSSD dataset, which contains 1000 images that have a relatively more complicated background. DUT-OMRON is a newly built dataset, which contains 5168 images that have a large variety not only in content but also in size with complicated background, thus, is the most difficult one. We choose these three datasets to evaluate the performance and robustness of our algorithm under different cases.

\subsection{Metrics}

We adopt the canonical precision-recall curve and F-measure to compare with other methods. Specifically, PR-curve is obtained by binarizing the predicted saliency map with a threshold varying from 0 to 255 , and F-measure is calculated with the formula given in [Ш]

$$
F_{\beta}=\frac{\left(1+\beta^{2}\right) \text { Precision } \times \text { Recall }}{\beta^{2} \text { Precision }+ \text { Recall }}
$$

where $\beta^{2}$ is set to 0.3 as done in [四, 四] to emphasize precision, and the given threshold is twice the mean value of a saliency map. Also, we use mean absolute error (MAE) to measure the difference between predicted saliency map and the corresponding binary ground truth.

\subsection{Performance}

In our experiments, we empirically set $n=200$ and $\lambda=0.1$, and parametric sensitivity analysis is given in the next section. PR curves, F-measure and MAE of all the eight methods on MSRA 1000, ECSSD and DUT-OMRON datasets are shown in Fig. 5. As mentioned before, MSRA1000 is the simplest dataset so that most methods perform well on it. Specifically, most methods achieve F-measure higher than 0.8, among which our method achieves the highest. ECSSD is more complicated than MSRA1000, so that performances of methods like SF [ $[\mathbf{Q}]$ and HC [G] drop heavily. While our method outperforms most methods except DRFI [ $[$ ] ]. DUT-OMRON dataset is the most complicated one, similar to the result on ECSSD, our methods still achieve a comparable result compared with the other methods. Note that MAE values of all methods except HC [6] on ECSSD and DUT-OMRON datasets are close to each other, approximately ranging between 0.15 and 0.20 .

As mentioned before, we consider salient object segmentation as a pre-processing step for high-level tasks, so it is expected to be efficient. Time-consumption of each method is given in Table. 1. We can see that even though DRFI [미 works best among all datasets, it takes about 10 seconds to test an input image, due to the feature-extraction on multi-level segmentation $[\square]$. HC $[$ [ $]$ is the most efficient, however, its performance is not good enough, as shown in Fig. 5. Among the rest methods, our algorithm takes the least time to achieve an excellent and also robust result.

To have an intuitive concept of the performance, we give a visual comparison of images chosen from the three datasets, and corresponding results are listed in Fig.6. As discussed 

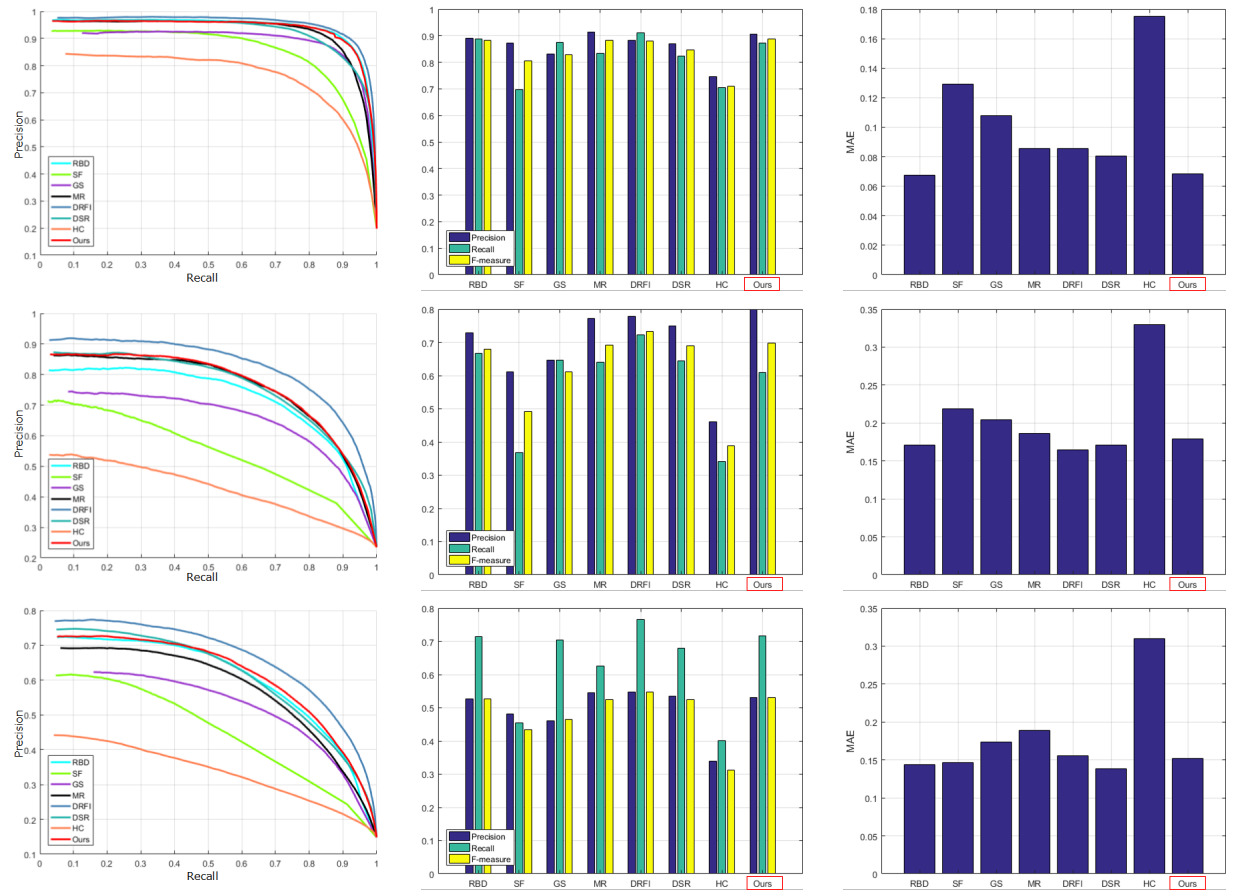

Figure 5: Comparison on Precision-Recall curves, F-measure and Mean Absolute Error (MAE) of eight methods on three benchmark datasets: from top to bottom are MSRA 1000, ECSSD and DUT-OMRON.

\begin{tabular}{c|c|c|c|c|c|c|c|c}
\hline Methods & RBD & SF & GS & MR & DRFI & DSR & HC & Ours \\
\hline Time(s) & 0.20 & 0.19 & 0.18 & 0.26 & 10 & 4.94 & 0.02 & 0.18 \\
\hline Code & Matlab & Matlab & Matlab & Matlab & Matlab & Matlab & Matlab & Matlab \\
\hline \multicolumn{6}{c|}{ Table 1: Time consumption of different methdos }
\end{tabular}

in Sect. 3.4, boundary connectivity [ $[\mathbf{G}]$ fails to determine the true background boundary when a frame exists around the content, as shown in the second image. The fourth image shows that our method is robust to multiple separate objects. Note that in the sixth image, the foreground has a lower lightness than background, so methods such as HC [ $[$ ] that use only global contrast will falsely take foreground as background. As only boundary prior is used in MR [ $[\mathrm{⿴囗}]$, the background is easily effected by detected foreground during diffusion, as the last row shows. Among the results of all methods, ours have the best uniformness.

\subsection{Parametric Sensitivity}

Our algorithm takes two parameters, the amount of superpixels $n$ and the weight coefficient of affinity $\lambda$. We examine the sensitivity to $F_{\beta}$ w.r.t. each parameter by fixing another one, as shown in Fig. 7. We can see that $F_{\beta}$ is relatively not sensitive to the amount of superpixels $n$ or weight coefficient $\lambda$. Therefore, it is flexible to segment input images into superpixels according to its size. A larger $n$ results in slightly higher $F_{\beta}$, while more computational cost. A smaller $\lambda$ promises better segmentation results, and effect of optimal compact seeds shows more importance in simple background than complex background. 


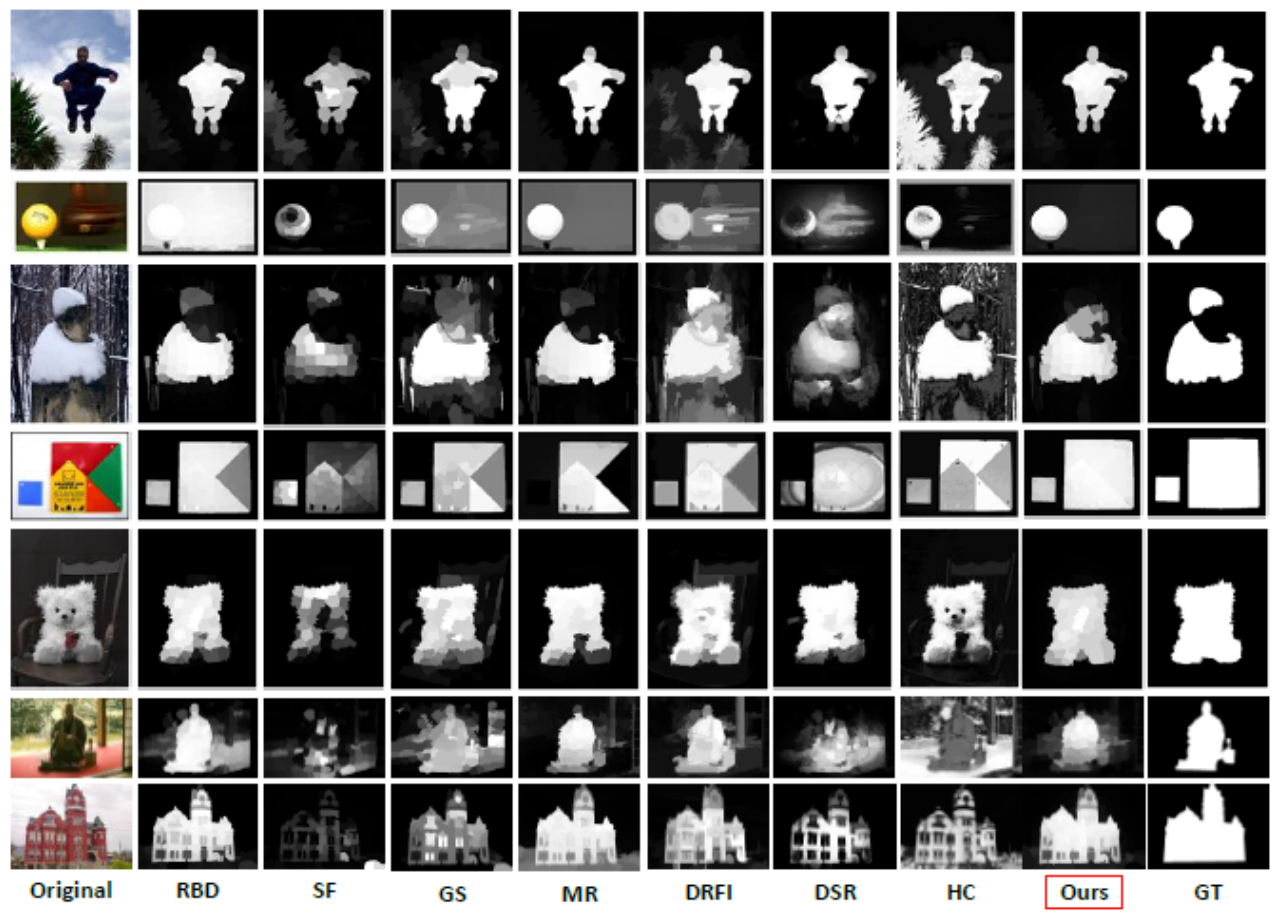

Figure 6: Saliency maps generated by different methods

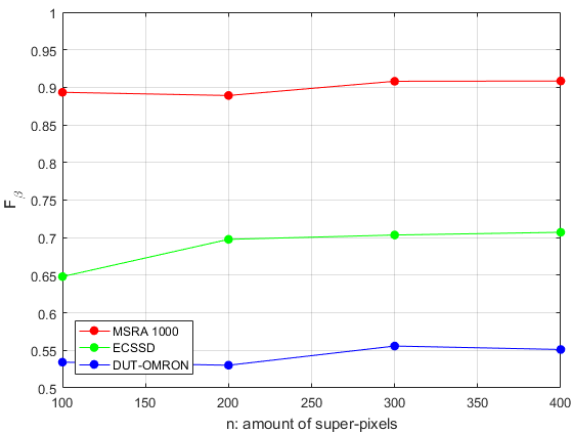

(a)

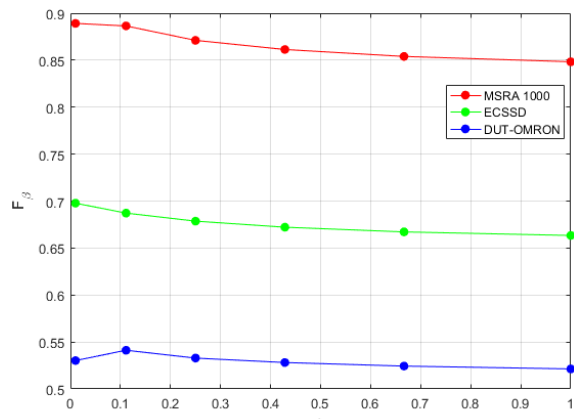

(b)

Figure 7: Parametric sensitivity analysis: (a) shows the variation of $F_{\beta}$ w.r.t. $n$ by fixing $\lambda=0.1$; (b) shows the variation of $F_{\beta}$ w.r.t. $\lambda$ by fixing $n=200$

\section{Conclusion}

This paper proposed a new framework for salient object segmentation via combination of compactness and boundary prior. Optimal seeds are set as those compact super-pixels and quadratic energy model is applied to diffuse compactness. Boundary prior is combined with the coarse saliency map generated by previous diffusion. We then re-apply the quadratic energy model to derive the final uniform saliency map. Experiments on three benchmark datasets have shown that our method achieves state-of-the-art result with high efficiency. 
Acknowledgement This work was supported partially by National Key Technology Research and Development Program of the Ministry of Science and Technology of China (No. 2015BAK36B00), in part by the Key Science and Technology of Shenzhen (No. CXZZ20150814155434903), in part by the Key Program for International S\&T Cooperation Projects of China (No. 2016YFE0121200), in part by the National Natural Science Foundation of China (No. 61571205).

\section{References}

[1] Radhakrishna Achanta, Sheila Hemami, Francisco Estrada, and Sabine Susstrunk. Frequency-tuned salient region detection. In Computer vision and pattern recognition, 2009. cvpr 2009. ieee conference on, pages 1597-1604. IEEE, 2009.

[2] Radhakrishna Achanta, Appu Shaji, Kevin Smith, Aurelien Lucchi, Pascal Fua, and Sabine Süsstrunk. Slic superpixels compared to state-of-the-art superpixel methods. IEEE transactions on pattern analysis and machine intelligence, 34(11):2274-2282, 2012.

[3] Ali Borji and Laurent Itti. State-of-the-art in visual attention modeling. IEEE transactions on pattern analysis and machine intelligence, 35(1):185-207, 2013.

[4] Ali Borji, Ming-Ming Cheng, Huaizu Jiang, and Jia Li. Salient object detection: A benchmark. IEEE Transactions on Image Processing, 24(12):5706-5722, 2015.

[5] Kai-Yueh Chang, Tyng-Luh Liu, Hwann-Tzong Chen, and Shang-Hong Lai. Fusing generic objectness and visual saliency for salient object detection. In Computer Vision (ICCV), 2011 IEEE International Conference on, pages 914-921. IEEE, 2011.

[6] Ming-Ming Cheng, Niloy J Mitra, Xiaolei Huang, Philip HS Torr, and Shi-Min Hu. Global contrast based salient region detection. IEEE Transactions on Pattern Analysis and Machine Intelligence, 37(3):569-582, 2015.

[7] Simone Frintrop, Erich Rome, and Henrik I Christensen. Computational visual attention systems and their cognitive foundations: A survey. ACM Transactions on Applied Perception (TAP), 7(1):6, 2010.

[8] Antonino Furnari, Giovanni Maria Farinella, and Sebastiano Battiato. An experimental analysis of saliency detection with respect to three saliency levels. In ECCV Workshops (3), pages 806-821, 2014.

[9] Ghurumuruhan Ganesan and Ye Li. Cooperative spectrum sensing in cognitive radio, part i: Two user networks. IEEE Transactions on wireless communications, 6(6):22042213, 2007.

[10] Stas Goferman, Lihi Zelnik-Manor, and Ayellet Tal. Context-aware saliency detection. IEEE Transactions on Pattern Analysis and Machine Intelligence, 34(10):1915-1926, 2012. 
[11] Mingming Gong, Kun Zhang, Tongliang Liu, Dacheng Tao, Clark Glymour, and Bernhard Schölkopf. Domain adaptation with conditional transferable components. In International Conference on Machine Learning, pages 2839-2848, 2016.

[12] Chenlei Guo and Liming Zhang. A novel multiresolution spatiotemporal saliency detection model and its applications in image and video compression. IEEE transactions on image processing, 19(1):185-198, 2010.

[13] Xiaodi Hou and Liqing Zhang. Saliency detection: A spectral residual approach. In Computer Vision and Pattern Recognition, 2007. CVPR'07. IEEE Conference on, pages 1-8. IEEE, 2007.

[14] Laurent Itti, Christof Koch, and Ernst Niebur. A model of saliency-based visual attention for rapid scene analysis. IEEE Transactions on pattern analysis and machine intelligence, 20(11):1254-1259, 1998.

[15] Bowen Jiang, Lihe Zhang, Huchuan Lu, Chuan Yang, and Ming-Hsuan Yang. Saliency detection via absorbing markov chain. In Proceedings of the IEEE International Conference on Computer Vision, pages 1665-1672, 2013.

[16] Huaizu Jiang, Jingdong Wang, Zejian Yuan, Yang Wu, Nanning Zheng, and Shipeng Li. Salient object detection: A discriminative regional feature integration approach. In Proceedings of the IEEE conference on computer vision and pattern recognition, pages 2083-2090, 2013.

[17] Christof Koch and Shimon Ullman. Shifts in selective visual attention: towards the underlying neural circuitry. In Matters of intelligence, pages 115-141. Springer, 1987.

[18] Vladlen Koltun. Efficient inference in fully connected crfs with gaussian edge potentials. Adv. Neural Inf. Process. Syst, 2(3):4, 2011.

[19] Jian Li, Martin D Levine, Xiangjing An, Xin Xu, and Hangen He. Visual saliency based on scale-space analysis in the frequency domain. IEEE transactions on pattern analysis and machine intelligence, 35(4):996-1010, 2013.

[20] Xiaohui Li, Huchuan Lu, Lihe Zhang, Xiang Ruan, and Ming-Hsuan Yang. Saliency detection via dense and sparse reconstruction. In Proceedings of the IEEE International Conference on Computer Vision, pages 2976-2983, 2013.

[21] Tie Liu, Zejian Yuan, Jian Sun, Jingdong Wang, Nanning Zheng, Xiaoou Tang, and Heung-Yeung Shum. Learning to detect a salient object. IEEE Transactions on Pattern analysis and machine intelligence, 33(2):353-367, 2011.

[22] Song Lu, Vijay Mahadevan, and Nuno Vasconcelos. Learning optimal seeds for diffusion-based salient object detection. In Proceedings of the IEEE Conference on Computer Vision and Pattern Recognition, pages 2790-2797, 2014.

[23] Long Mai, Yuzhen Niu, and Feng Liu. Saliency aggregation: A data-driven approach. In Proceedings of the IEEE Conference on Computer Vision and Pattern Recognition, pages 1131-1138, 2013. 
[24] Federico Perazzi, Philipp Krähenbühl, Yael Pritch, and Alexander Hornung. Saliency filters: Contrast based filtering for salient region detection. In Computer Vision and Pattern Recognition (CVPR), 2012 IEEE Conference on, pages 733-740. IEEE, 2012.

[25] Christian Siagian and Laurent Itti. Biologically inspired mobile robot vision localization. IEEE Transactions on Robotics, 25(4):861-873, 2009.

[26] Ulrike Von Luxburg. A tutorial on spectral clustering. Statistics and computing, 17(4): 395-416, 2007.

[27] Qiaosong Wang, Wen Zheng, and Robinson Piramuthu. Grab: Visual saliency via novel graph model and background priors. In Proceedings of the IEEE Conference on Computer Vision and Pattern Recognition, pages 535-543, 2016.

[28] Yichen Wei, Fang Wen, Wangjiang Zhu, and Jian Sun. Geodesic saliency using background priors. Computer Vision-ECCV 2012, pages 29-42, 2012.

[29] Qiong Yan, Li Xu, Jianping Shi, and Jiaya Jia. Hierarchical saliency detection. In Proceedings of the IEEE Conference on Computer Vision and Pattern Recognition, pages 1155-1162, 2013.

[30] Chuan Yang, Lihe Zhang, Huchuan Lu, Xiang Ruan, and Ming-Hsuan Yang. Saliency detection via graph-based manifold ranking. In Proceedings of the IEEE conference on computer vision and pattern recognition, pages 3166-3173, 2013.

[31] Wei Yuan, Xinge You, Jing Xu, Henry Leung, Tianhang Zhang, and Chun Lung Philip Chen. Multiobjective optimization of linear cooperative spectrum sensing: Pareto solutions and refinement. IEEE transactions on cybernetics, 46(1):96-108, 2016.

[32] Dingwen Zhang, Junwei Han, Jungong Han, and Ling Shao. Cosaliency detection based on intrasaliency prior transfer and deep intersaliency mining. IEEE transactions on neural networks and learning systems, 27(6):1163-1176, 2016.

[33] Wangjiang Zhu, Shuang Liang, Yichen Wei, and Jian Sun. Saliency optimization from robust background detection. In Proceedings of the IEEE conference on computer vision and pattern recognition, pages 2814-2821, 2014. 